\title{
Análisis del Índice de Riesgo de la Calidad del Agua para Consumo Humano -Irca- y su Relación con Variables Meteorológicas y Ubicación Geográfica para el Departamento del Tolima en los Años $2012-2013$ \\ Analysis Of the Risk IndeX of the Water Quality for Human Consumption -IRCA- and ITS Relation WITH Meteorological Variables and Geographical Location FOR THE TOLIMA DePARTMENT IN the YeARS 2012 - 2013
}

1Víctor Leonardo López Jiménez, ²Judy Johanna Martínez Ramos, ${ }^{3}$ Diana Paola Almario Guio

Ingeniería Ambiental y Sanitaria, Universidad de la Salle, Bogotá, Colombia,

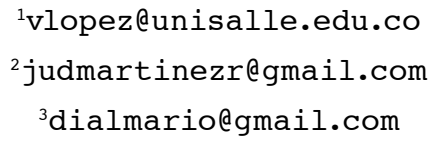

Recibido: 10/08/2015 • Aprobado: 12/11/2015

\section{RESUMEN}

Este proyecto se desarrolló con el fin de contribuir a la gestión de mejora continua en el proceso de vigilancia y control de la calidad del agua para consumo humano en el departamento del Tolima. A través del análisis de la información reportada por el Sistema de Información para la Vigilancia de la Calidad del Agua para Consumo Humano, Sivicap, con el coeficiente de Pearson como herramienta estadística, se determinaron las correlaciones entre parámetros que componen el IRCA, para evidenciar las posibles mejoras acerca del cálculo de este índice y su planteamiento. Se estableció la relación entre variables meteorológicas con el IRCA, consolidando estas en mapas temáticos, lo cual permite un análisis más detallado del mismo por municipio, con base en las condiciones geográficas del área de estudio. Finalmente, se formularon alternativas de mejoramiento dirigidas a las acciones de control y vigilancia de la calidad del agua para consumo humano. De esta manera, se identificaron las inconsistencias de la información reportada por el Sivicap, que refiere principalmente al incumplimiento de la Resolución 2115 de 2007, en relación con los parámetros de medición de las características físicas, químicas y microbiológicas; además, se estableció la alteración del índice por factores externos como el clima, aumentando su resultado en presencia de fenómenos de precipitación y temperatura pronunciados. El resultado de este estudio, conlleva a determinar los factores que intervienen en la variación del índice, proporcionando así, información pertinente que puede aplicarse como argumento para la toma de decisiones y gestión de los entes responsables de la calidad del agua.

Palabras clave: agua potable, calidad del agua, condiciones meteorológicas, IRCA, ubicación geográfica. 


\section{Abstract}

This project was developed in order to contribute to the management of continuous improvement in the process of monitoring and control of the water quality for human consumption, in the department of Tolima. Through the analysis of the information reported by the information system for monitoring the quality of water for human consumption, Sivicap, with the Pearson coefficient as statistical tool, the correlations between parameters which are part of the IRCA were determined, to demonstrate the possible improvements regarding the calculation of this index and its approach. The relationship between weather variables and the IRCA was established, consolidating these in thematic maps, which allows a more detailed analysis of the same, by municipality, on the basis of geographical conditions in the study area. Finally, alternatives of improvement were formulated and addressed to the actions of control and surveillance of the water quality for human consumption. In this way, inconsistencies in the information reported by the SIVICAP were found, which mainly refer to non-compliance of the resolution 2115 of 2007, related to the measurement parameters of physical, chemical and microbiological characteristics; In addition, alteration of the index by external factors, such as weather, was established, increasing its result in the presence of pronounced phenomena of precipitation and temperature. The result of this study, leads to determine the factors involved in the variation of the index, thus, providing relevant information that can be applied as an argument for decision-making and management of entities responsible for the water quality.

Keywords: drinking water, geographical location, IRCA, meteorological conditions, water quality.

\section{INTRODUCCIÓN}

La calidad del agua para consumo humano es un factor determinante en la salud pública; en Colombia, las deficientes condiciones sanitarias asociadas a la pobreza y al deterioro ambiental, al igual que las falencias en la prestación del servicio y la falta de cobertura del mismo, ha generado que en zonas urbanas alejadas o en zonas rurales se incremente el porcentaje de enfermedades causadas por el consumo de agua contaminada [1]. Por tal razón, con el fin de monitorear, prevenir y controlar los riesgos sobre la salud humana, se establece el Sistema para la Protección y Control de la Calidad del Agua para Consumo Humano con el Decreto 1575 de 2007, el cual define los instrumentos, así como las funciones y acciones, que permiten una gestión coordinada entre los responsables de la garantía de la calidad del agua, destacando las acciones de control de las personas prestadoras, la función de vigilancia de las autoridades sanitarias de los departamentos y la gestión de reglamentación y diseño de protocolos de los Ministerios de Salud y Protección Social, y de Ambiente y Desarrollo Sostenible, incluyendo al Instituto Nacional de Salud, el cual ha desarrollado el aplicativo Sivicap, que permite a todas las autoridades sanitarias departamentales reportar los datos de la vigilancia de la calidad del agua en función de sus actividades de inspección, vigilancia y control en el país [2].

La importancia de la calidad del agua en Colombia se ha convertido en una temática con mayor relevancia en la última década, de tal manera, que el acceso a agua potable y el saneamiento han aumentado significativamente en comparación con años anteriores. En ese contexto, se han implementado en el país programas que permitan mejorar tanto la calidad del servicio de abastecimiento como la cobertura en las zonas rurales [3]. Como iniciativa, entonces, se crea el Índice de Riesgo de la Calidad de Agua para consumo humano, el 
cual, con base en características físico-químicas y microbiológicas del agua, determina el riesgo en la salud humana [4]. Este indicador es tenido en cuenta tanto por entidades nacionales como internacionales para establecer la calidad de agua que consume la población en todo el territorio nacional. Al tratarse de un indicador ampliamente acogido, sobre él se generan informes nacionales, departamentales y municipales, en los cuales se expone el riesgo del agua potable; como consecuencia y buscando dar cumplimiento a la normatividad, se incluyen en los proyectos de desarrollo departamentales y municipales, planes con acciones futuras para el mejoramiento del índice $y$, por ende, de la calidad del agua abastecida.

Para el departamento del Tolima, el tema de los servicios públicos es un componente fundamental para mejorar las condiciones de vida de los habitantes; por esto, se plantean una serie de estrategias en procura de lograr coberturas óptimas, pero, adicionalmente, con calidad, eficiencia, eficacia y continuidad en la prestación de los servicios. En el caso del agua potable y saneamiento básico, el objetivo es disminuir el IRCA; que el nivel de riesgo correspondiente a los años de estudio baje de manera continua hasta alcanzar un nivel sin riesgo, es decir, por debajo del 10\% del índice de riesgo y, por tanto, reducir los casos de morbilidad y mortalidad de la población [5]. En ese marco, la correcta información sobre la calidad del agua, utilizando el IRCA como instrumento base, es fundamental para el desarrollo del departamento y conjuntamente del país; en tal sentido, la magnitud e importancia del índice se convierte en una temática atrayente. En el proceso se dispone de la información obtenida de la vigilancia de las autoridades sanitarias reportada en el Sivicap, la cual involucra factores internos comprendidos para el cálculo del índice. La aplicación de la herramienta carece hoy en día de protocolos que permitan garantizar la consistencia de los datos y muestra debilidad en la información específica relacionada con factores externos que pueden afectar el resultado del índice y que permitan evidenciar de forma integral la realidad referente a la calidad del agua en los municipios, departamentos y regiones del país. Como consecuencia de lo anterior, el objetivo de este estudio se centró en analizar el IRCA, el comportamiento de las variables que lo componen y su relación con el régimen de precipitación y temperatura dentro del área geográfica del departamento del Tolima en el periodo $2012-2013$.

\section{Metodología}

Para el desarrollo del estudio se realizó un análisis cualitativo y cuantitativo, que permitiera establecer causa-efecto entre los elementos y fenómenos estudiados mediante la interpretación de la información. El enfoque cuantitativo permitió ejecutar a través de métodos estadísticos las correlaciones entre variables numéricas referentes a los datos reportados del IRCA y las variables meteorológicas determinadas en el estudio. Con el análisis cualitativo, respecto del planteamiento de las correlaciones, se logró un diagnóstico de tipo descriptivo en el cual se especificaron las propiedades, características y rasgos importantes relacionados, con el fin de establecer tendencias o acciones predominantes en el manejo de la información tratada.

Se utilizó como base el consolidado municipal del IRCA para el departamento del Tolima en los años 2012 y 2013, obtenida de la base de datos del subsistema Sivicap y de la información meteorológica del Ideam; paso seguido, se definieron e implementaron criterios para seleccionar y verificar la información necesaria para cada una de las etapas que componen el análisis estadístico, todo con el fin de obtener los mejores resultados al final de estudio.

\section{A. Criterios de selección}

Inicialmente, se definió la variable independiente por correlacionar con los demás parámetros del IRCA, incluyendo como criterio base los cuatro parámetros más representativos para el cálculo del mismo; es decir, los que tienen mayor puntaje según 
la resolución 2115 de 2007: turbiedad, cloro residual libre, coliformes totales y Escherichia coli; estos dos últimos presentaron datos que no son aplicables para el análisis estadístico por medio del Coeficiente de Pearson y fueron descartados tanto para la selección como variable independiente, como para la utilización de estos en las correlaciones. Se escogió la turbiedad como variable independiente, pues, en comparación con cloro residual libre, fue el parámetro con mayor cantidad de datos disponibles que permitió arrojar un estudio estadístico de mayor viabilidad y representatividad, además de ser uno de los parámetros del IRCA con mayor frecuencia de medición, que puede ser utilizado como indicador básico de calidad del agua potable.

Se utilizó como base la cantidad de muestras disponibles reportadas por el Sivicap en el ámbito departamental. Una vez verificada la información, se dispuso de 790 muestras para el cálculo del IRCA en 2012 y de 890 muestras para 2013, incluyendo exclusivamente los municipios que disponían de cinco muestras o más; posteriormente, se eligieron los parámetros que tenían tres o más datos respecto de la variable independiente, obteniendo para analizar 14 parámetros para el año 2012, incluyendo alcalinidad, calcio, cloruros, cloro residual libre, color, dureza total, fosfatos, hierro total, magnesio, nitratos, nitritos, $\mathrm{pH}$, sulfatos y turbiedad. Adicionalmente a estos parámetros, se seleccionó aluminio, resultando 15 parámetros para el desarrollo de las correlaciones para el año 2013.

En la selección de datos para el cálculo del IRCA se incluyeron únicamente los municipios con información mayor o igual a seis meses, resaltando así 16 municipios analizados en 2012 y 25 municipios en el año 2013. Adicionalmente, para la selección de datos para correlaciones con variables meteorológicas, se tomó la información de estaciones referidas en el Atlas Climatológico de Colombia e información meteorológica del catálogo de estaciones meteorológicas del Ideam; solo se analizaron los 16 municipios donde operan estaciones meteorológicas que reportan información para los años de estudio (2012-2013) y que por su ubicación geográfica permitieron una correlación con la información arrojada por el IRCA; de este modo, tan solo 16 municipios para el 2012 y 10 para el 2013 fueron relacionados estadísticamente.

Utilizando el programa Microsoft Exce ${ }^{\circledR}$ como herramienta de análisis de datos, se determinó en el ámbito municipal, la relación entre parámetros del IRCA en cada año y entre las variables meteorológicas con el IRCA mensual, usando como herramienta el coeficiente de correlación de Pearson. Posteriormente, se clasificaron los coeficientes obtenidos con base en correlación fuerte, moderada, débil y ninguna [6]. Por otra parte y como elemento fundamental, se determinó la significación del coeficiente de correlación, para establecer si las variables estaban relacionadas en realidad o tan solo presentaban dicha relación como consecuencia del azar, enfatizando en el análisis de correlaciones fuertes.

\section{B. Análisis estadístico entre parámetros del IRCA}

Como acción principal, se calculó el IRCA mensual en cada municipio a partir de la Ecuación 1, establecida en el artículo 14 de la Resolución 2115, lo cual se complementó con el cálculo del IRCA promedio ponderado anual para el departamento, municipios y personas prestadoras utilizando la Ecuación 2.

$$
\operatorname{IRCA}(\%)=\frac{\sum \operatorname{IRCA}_{\mathrm{m}}}{\mathrm{N}_{\mathrm{m}_{\mathrm{i}}}} \times 100
$$

$$
\operatorname{IRCA}_{\mathrm{ppa}}=\frac{\sum_{\mathrm{i}=1}^{12}\left(\mathrm{IRCA}_{\mathrm{pm}_{\mathrm{i}}} * \mathrm{~N}_{\mathrm{m}_{\mathrm{i}}}\right)}{\mathrm{N}_{\mathrm{t}_{\mathrm{i}}}}
$$

\section{Donde}

IRCA ${ }_{m}$ : IRCA de cada muestra realizada en el mes. $I_{\mathrm{RCA}}$ : IRCA promedio ponderado anual.

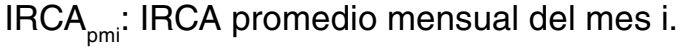

$\mathrm{N}_{\mathrm{mi}}$ : Número total de muestras tomadas en el mes i. $\mathrm{N}_{\mathrm{ti}}$ : Número total de muestras tomadas en el año. 
Se definió la variable independiente por correlacionar con los demás parámetros del IRCA a partir de los criterios de selección; luego, se calculó el Coeficiente de Pearson, clasificando los coeficientes obtenidos y determinando la significación del coeficiente para determinar las correlaciones entre los parámetros que componen el IRCA en cada municipio. Para concluir esta etapa, se analizaron los resultados obtenidos, comparando el índice en los dos años de estudio y determinando los componentes que afectan su resultado.

\section{Análisis estadístico entre variables meteorológi- cas y condiciones físico-geográficas con el IRCA}

Se estableció por medio del coeficiente de Pearson la correlación entre el IRCA mensual obtenido en la etapa anterior con las variables meteorológicas, precipitación y temperatura, para los meses con datos disponibles en los municipios previamente seleccionados; en atención a esto, de acuerdo con la fuerza de correlación obtenida y la significación del coeficiente, se analizó la relación resultante. Además del análisis estadístico, se desarrolló un análisis teórico de la influencia de las condiciones físico-geográficas del área del estudio en el comportamiento del IRCA. De todo esto, se desprendió un análisis amplio referente a las inconsistencias y falencias determinadas, que integró la calidad de la información con la cual se trabajó, la relación entre los parámetros propuestos y la fortaleza del índice frente a la realidad de la calidad del agua potable en el área de estudio; en consecuencia, se formularon alternativas de mejoramiento dirigidas a las acciones de vigilancia y control de la calidad del agua para consumo humano.

Para concluir, utilizando ARCGIS $\AA$ como sistema de información geográfica se elaboraron mapas temáticos referentes a la ubicación de puntos de muestreo y distribución mensual de los valores del IRCA para los municipios analizados, dando una muestra de lo que se propone para un análisis detallado y puntual del IRCA; para la estructuración de los mapas se referenció el año 2013, considerando la disponibilidad de información en los años de estudio. Se concluyó que más del $80 \%$ de las muestras reportadas por Sivicap no precisaban coordenadas geográficas que permitieran una ubicación exacta de los puntos; fueron ubicadas, entonces, en áreas cercanas con base en descripciones generales como el lugar y la dirección de cada punto.

\section{Resultados y Discusión}

\section{A. Análisis estadístico del IRCA}

Se evidenció la baja calidad de datos frente a las muestras reportadas. Debido a que se encontraron muestras sin reporte de resultados, muestras repetidas, con insuficiente medición de parámetros e incoherencia de datos, se excluyeron del análisis del IRCA, pues alteraban significativamente su resultado, disminuyendo así la cantidad de muestras y datos utilizados para cada una de las etapas. Aun así, con la turbiedad como variable independiente, se obtuvieron las correlaciones más significativas con hiero y color aparente total en los dos años de estudio.

Utilizando el municipio de lbagué como ejemplo de las correlaciones, debido a la mayor cantidad de muestras, se presenta el diagrama de dispersión en la Fig. 1 con su respectiva regresión lineal para los años 2012 y 2013, el cual permite calcular el coeficiente de Pearson y determinar, según su significación, la relación existente entre la turbiedad y los demás parámetros seleccionados. De este análisis se obtuvo que la turbiedad se correlaciona moderadamente con el color aparente en los dos años; igualmente, se correlaciona moderadamente con el hierro total en el año 2012. 

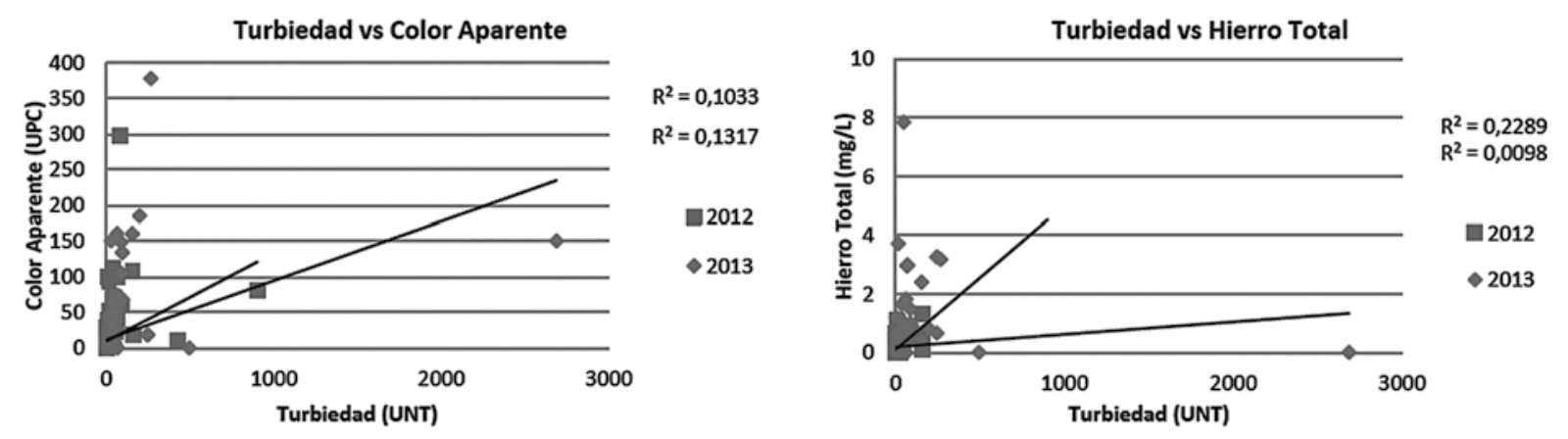

Fig. 1 Regresión lineal para correlaciones entre turbiedad con color aparente (Izq.) y turbiedad con hierro total (Der.) para los años 2012 y 2013, Ibagué.

Por otra parte, el municipio de Armero se encontraba entre los municipios de baja representatividad con relación a las muestras reportadas, por lo cual se utilizó para contrastar con los resultados obtenidos en el municipio de lbagué; se destaca que la turbiedad se correlacionó fuertemente con hierro total y color aparente en los dos años.

Con base en los datos suministrados por el Sivicap, el cual no reporta información para los municipios de Ambalema y Planadas en el año 2012, se obtuvo el IRCA promedio anual departamental de $33,31 \%$ y $27,45 \%$ para los años 2012 y 2013, respectivamente, correspondientes a nivel de riesgo medio, comparándolo con el resultado del IRCA calculado a partir de los datos depurados y seleccionados, el cual correspondió a 41,26\% para el 2012 y a $37,55 \%$ para el 2013 , relativos a nivel de riesgo alto en ambos años. Esto determinó que efectivamente existen muestras con información inconsistente en el reporte del Sivicap que alteran significativamente el resultado del IRCA.

Es de anotar, que para verificar el cumplimiento de la norma respecto de la frecuencia y el número de muestras de vigilancia de la calidad física y química del agua para consumo humano que debe realizar la autoridad sanitaria en la red de distribución, es necesario conocer el número de habitantes atendidos por persona prestadora por municipio, como lo refiere el Artículo 24 de la Resolución 2115 de 2007, lo cual no se especifica en el reporte; además, la norma no contempla la medición de todos los parámetros en una muestra, pues la frecuencia de medición se exige dependiendo de la característica, alterando de esta manera el resultado del IRCA. Aun así, según el número de muestras tomadas en cada mes para cada municipio, y basándose en el mínimo número de habitantes atendidos por persona prestadora ( $\leq 2.500$ habitantes), se deduce que en general en el departamento no se cumple la norma en cuanto a la frecuencia mínima de muestras por analizar que corresponde a una muestra bimestral en el análisis de turbiedad, color aparente, $\mathrm{pH}$ y cloro residual libre, pues el mayor porcentaje de municipios no reporta información completa, a excepción de los municipios de Cajamarca y Mariquita para el año 2012 y Cajamarca, Anzoátegui y Rovira para el año 2013, los cuales corresponden al $4,44 \%$ del total de municipios que reporta información en el 2012 y al 6,38\% en el 2013, cifra poco representativa para determinar un IRCA promedio anual en el departamento, influyendo en la comparación del IRCA en cada municipio; adicionalmente, no se tienen en cuenta los mismos meses para el cálculo del índice en los dos años.

Comparando el nivel de riesgo entre municipios asimiles donde se calculó el IRCA promedio anual en los dos años, se reveló un comportamiento similar, excepto en Villahermosa donde se registró un nivel de riesgo alto para 2012 y en el siguiente año se presentó sin riesgo como se observa en la Fig. 2. Sin embargo, la distribución de los puntos de muestreo no registró puntos fijos para los dos años, estableciendo que la ubicación de estos se selecciona de forma aleatoria 
en cada año, lo cual influye en el resultado del IRCA y no permite realizar un análisis comparativo y de evolución que describa el IRCA para diferentes años. Más aún, el tipo de agua distribuida también influye en el comportamiento del índice, caracterizado por un mayor valor en el agua sin tratamiento, donde el IRCA calculado en el agua tratada correspondió a nivel de riesgo medio y bajo para 2012 y 2013 respectivamente; para el agua sin tratamiento se obtuvo un IRCA con nivel de riesgo alto en los dos años.

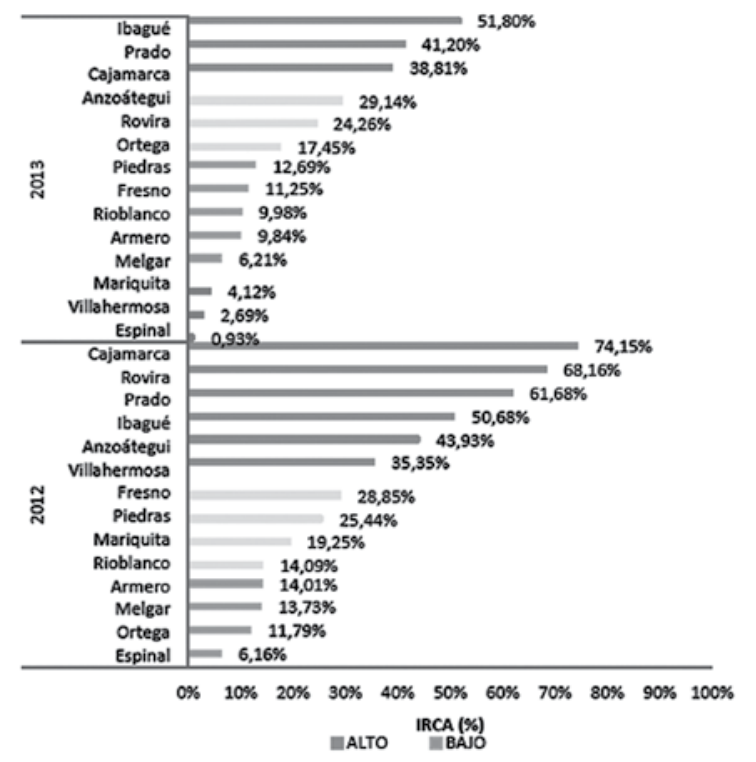

Fig. 2 Comparación del nivel de riesgo para municipios asimiles en los años 2012 y 2013.

\section{B. Análisis entre variables meteorológicas y con- diciones físico-geográficas con el IRCA}

La mayoría de los puntos se ubicaron en las zonas montañosas, con mayor concentración en los centros poblados, especialmente en la ciudad de lbagué,

IRCA mensual vs Precipitación

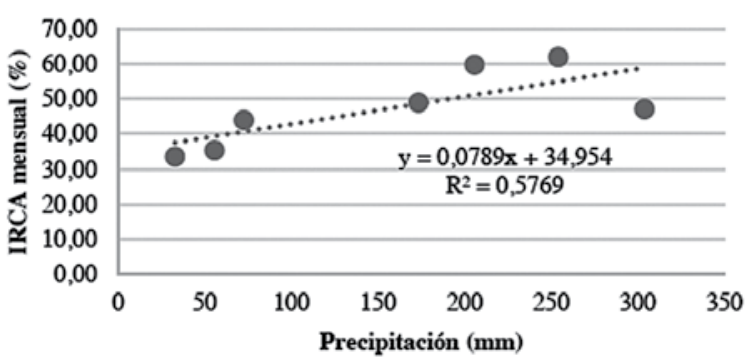

destacando que la mayor cantidad de puntos de muestreo con nivel de riesgo alto se distribuyó aleatoriamente en el centro del departamento; consecuentemente, la inviabilidad sanitaria se encontró en zonas rurales cuyos puntos de muestreo se reconocieron cercanos a los cuerpos de agua, lo que permitió suponer una distribución del agua sin tratamiento previo.

Se destacaron correlaciones fuertes para el año 2012 entre el IRCA y la precipitación en los municipios de Ibagué, Piedras, Rioblanco y Villahermosa, y solo en Ibagué existió relación entre la temperatura y la calidad del agua. Se aclara que no se encontraron relaciones fuertes entre las variables y el IRCA para el año 2013. Adicionalmente, factores como la falta de información meteorológica para los años de estudio, la pobreza de la red y la falta de protocolos para el monitoreo de los parámetros que componen el índice incidieron en la obtención de un análisis estadístico limitado.

Las relaciones entre la precipitación con el IRCA establecidas en los municipios de lbagué, Piedras y Rioblanco fueron directamente proporcionales como se puede apreciar en la Fig. 3. De esta manera, se calificó acorde con el régimen bimodal de la zona, pues, en la mayoría de los casos, en los meses más lluviosos, el valor del IRCA aumentó. Por otra parte, no se establecieron relaciones representativas para identificar la temperatura como un factor determinante en la calidad del agua para consumo humano relacionado con las fuentes de abastecimiento; únicamente se trazó relación inversamente proporcional en el municipio de lbagué.

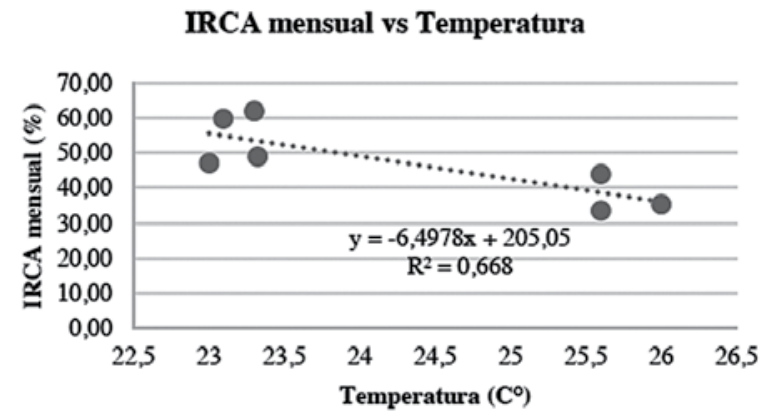

Fig. 3 Correlación de Pearson entre IRCA mensual vs Precipitación mensual (Izq.) e IRCA mensual vs Temperatura (Der.) para el municipio de Ibagué en el año 2012. 
La importancia de la precipitación se da en muchos aspectos relacionados con el abastecimiento de agua, ya que las fuentes de abastecimiento, como ríos, embalses y pozos, son alimentadas directa o indirectamente por la precipitación [7]. En relación con esto, se destaca que la calidad del agua puede estar influenciada por la alteración del suelo, la degradación ambiental alrededor de las cuencas, la remoción de material y el uso de productos químicos como plaguicidas y fertilizantes, producto de las actividades económicas que se desarrollan en el municipio. La agricultura es considerada una de las principales fuentes contaminantes de los cuerpos de agua y, junto con la ganadería, se constituye en el principal factor de cambio de uso del suelo. Es decir, el despojo de la cobertura vegetal nativa, como consecuencia de la capacidad de almacenamiento de los suelos, decrece significativamente y, por tanto, la escorrentía superficial aumenta; un cambio constante de uso del suelo puede llegar a erosionarlo y bajo un evento de precipitación aumenta el arrastre de material [8].

En los municipios de Ibagué y Vistahermosa se registraron altos niveles de riesgo, seguramente, debido a factores asociados a la actividad minera que se ejecuta en la vertiente oriental de la cordillera Central donde se encuentran ubicados [9], zona caracterizada por altas precipitaciones anuales. No obstante, no se evidenció este comportamiento en el municipio de Rioblanco, pues allí, solo se registraron datos de un mismo punto de muestreo (quebrada El Quebradón). Piedras, por el contrario, es un municipio con bajas precipitaciones a lo largo del año y que presenta un paisaje de valle en los alrededores del río Opia, excluyéndolo además de actividades mineras [10]; aun así, se relacionó con la precipitación de manera directa y en los últimos meses, en los que la precipitación se mantuvo constante, el índice también lo hizo, con niveles de riesgo más bajos en comparación con los otros municipios.

Por lo mencionado anteriormente y como se aprecia en la Fig. 4, la turbiedad fue el principal parámetro alterado por el incremento de la lluvia, aumentando a medida que la precipitación incrementaba; no obstante, también se puede presentar alteración de las características químicas del agua debido al tamaño de las partículas de los sedimentos y al volumen del carbono orgánico en partículas asociadas con los sedimentos.

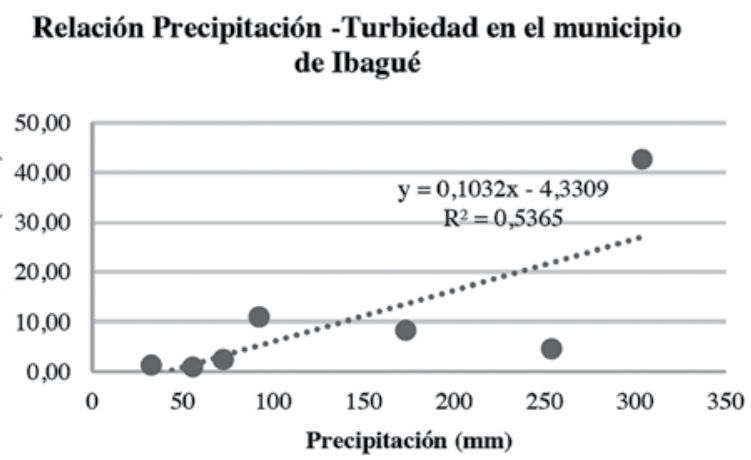

Fig. 4 Relación entre la precipitación y la turbiedad para el departamento de Ibagué en el año 2012.

Como se identifica en la Fig. 5, los picos más altos del IRCA se expusieron en los periodos de lluvia en el municipio de Rioblanco; de igual forma, como se reflejó en Ibagué y Piedras, en efecto, se encontró acorde con el comportamiento del régimen bimodal, aclarando que el $100 \%$ de la precipitación correspondía al total de la precipitación originada en los meses sobre los cuales se realizó el contraste con el IRCA.

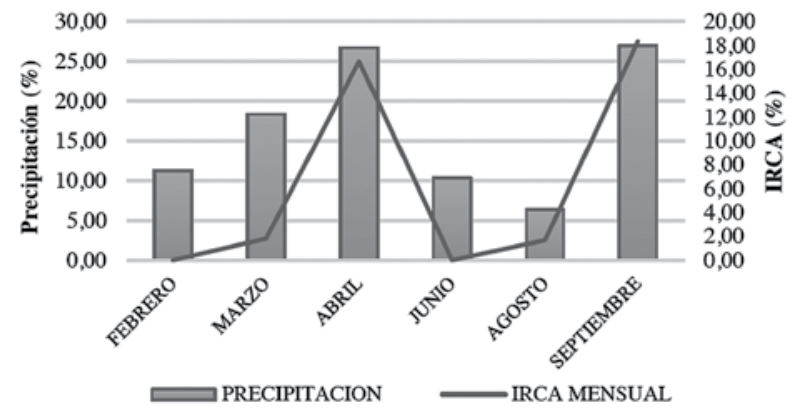

Fig. 5 Distribución mensual de la Precipitación y del IRCA mensual en el municipio Rio blanco, año 2012.

Por último, la mayor concentración de los puntos de muestreo se presentó en la ciudad de lbagué con rangos de riesgo muy variables: desde inexistentes hasta inviables sanitariamente. Además, se estableció que 
la mayoría de puntos con niveles de riesgo alto se ubicaron en zonas montañosas del departamento, ya que $85 \%$ de estos puntos se encontraban en la vertiente oriental de la cordillera Central como se establece en la Fig. 6. Esta zona cuenta con un paisaje de montaña con variabilidad climática [11], desde el piedemonte, donde se ubicaron los puntos de muestreo de la capital ibaguereña, hasta piso páramo en puntos de muestreo en el municipio de Cajamarca. Los puntos más altos se encontraron próximos al volcán Machín con una altura de $3.550 \mathrm{msnm}$ y al Nevado del Tolima con alturas cercanas a los 3.000 msnm; los puntos en la parte baja de la montaña se ubicaron a una altura alrededor de los $1.000 \mathrm{msnm}$.

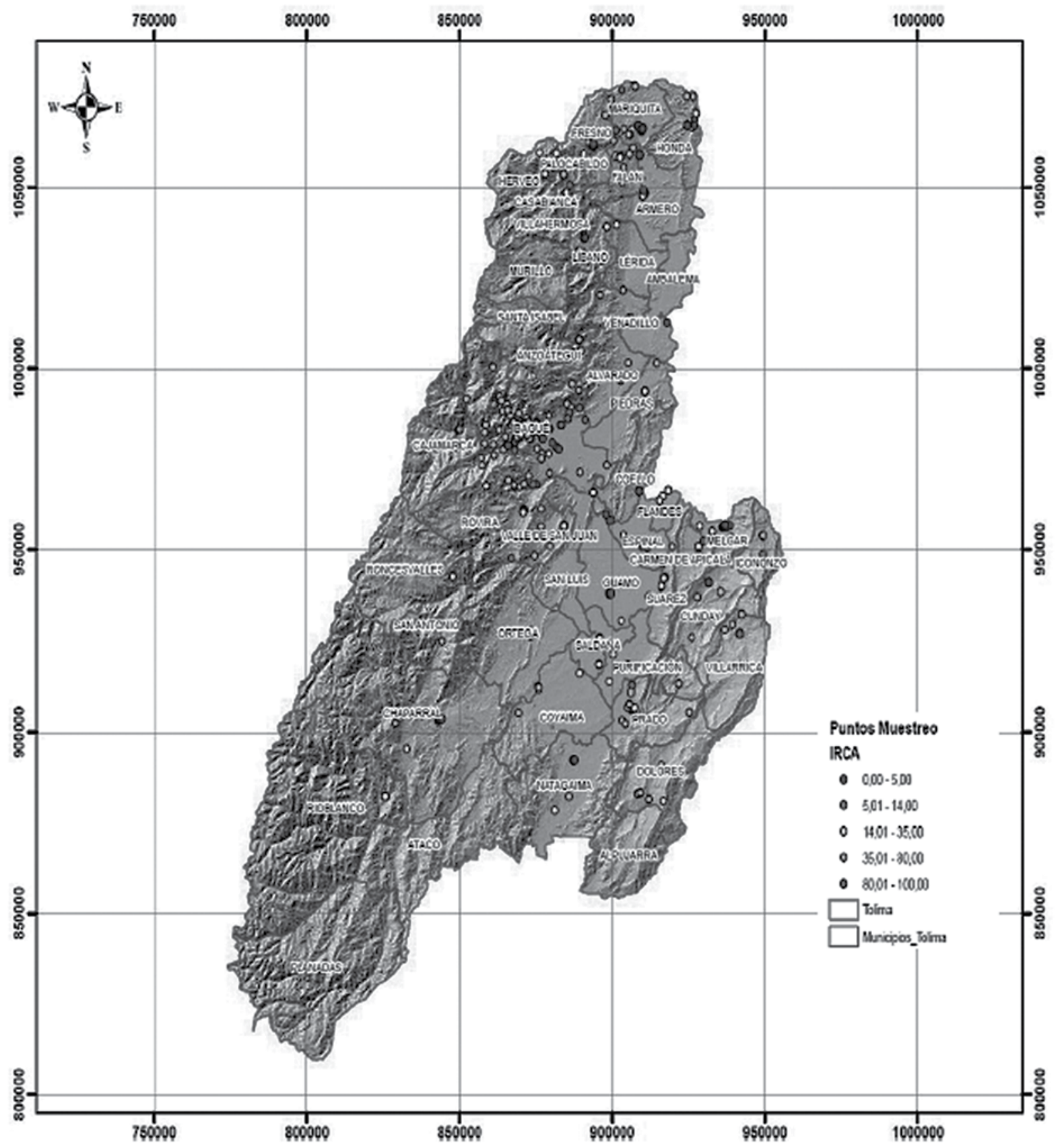

Fig. 6 Mapa de ubicación de puntos de muestreo según descripción del SIVICAP en el año 2013. 
La alteración de los parámetros considerados en el análisis puede estar relacionada con el cambio extremo de uso del suelo que se ha venido evidenciando en el departamento en los últimos años, además de las actividades mineras desarrolladas sobre la cordillera Central, propiamente en el paisaje de montaña. Teniendo en cuenta que la extracción del carbón, del oro y de otros materiales y metales generan alteraciones directas en los suelos, se asume que hay una remoción y deposición del material tanto en el suelo como en el aire y que asimismo se desencadena un descapote de la cobertura vegetal [12]. Este tipo de relieve con pendientes acentuadas y una escorrentía fuertemente pronunciada por la acción de la gravedad, permite un arrastre de dichos materiales producto de la actividad minera. Bajo ese concepto, los valores más altos del IRCA pueden relacionarse con un lavado de contaminantes que son conducidos desde lo más alto de la montaña aguas arriba de los cuerpos de agua hasta centros poblados que quedan en la parte baja 0 en paisaje de piedemonte.

El $42 \%$ de los puntos que reportaron agua inviable sanitariamente, se establecieron cercanos e incluso aledaños a cauces de agua; un $43 \%$ de puntos se aglomeró en el municipio de lbagué específicamente en la comuna trece a las afueras del casco urbano, puntos pertenecientes a barrios de invasión al sur que se han venido expandiendo y urbanizando y el $15 \%$ restante fueron puntos aleatoriamente distribuidos que no obedecen a ninguna tendencia hacia una característica fisiográfica.

En concordancia con lo comentado, las fuentes abastecedoras donde se ubicaron cercanos puntos de muestreo pueden afectarse por las diversas actividades desarrolladas en el departamento. También se resaltó la ubicación de Hidroeléctricas en Chaparral, Rioblanco y Roncesvalles, las cuales pueden afectar directamente los flujos de los cauces de agua; esta alteración puede traducirse en un incremento de transporte de material particulado y la degradación ambiental. Por otro lado, cerca al embalse de Prado se hallaron puntos comprendidos en el distrito de riego del Tolima, alteraciones que se reflejan en los cuerpos de agua, tanto principales como secundarios. Los ríos alterados bajo la variación de sus cauces son, el río Magdalena, el río Cuinde, río Cucuana, río Combeima, río Alvarado y río Guali.

Se pudo establecer que la inequidad en los puntos de muestreo entre la zona urbana y rural se refleja espacialmente al momento de mostrar la distribución del nivel de riesgo en el territorio. Una vez revelados los casos más críticos del nivel de riesgo del agua en función de características fisiográficas, se hizo alusión a los riesgos medios y a la calidad de agua sin riesgo, cuyas muestras se distribuyeron $97 \%$ en zonas urbanas y el $3 \%$ restante en puntos tomados en la zona rural, de las cuales el $85 \%$ pertenece a viviendas aledañas a vías principales y secundarias. Todas estas razones permiten argumentar una falta de cobertura con respecto a la vigilancia de la calidad de agua para poblaciones alejadas a los centros urbanos. Con esto, se argumenta que el resultado del IRCA en el ámbito departamental, está dado por una baja cobertura territorial, con énfasis en las cabeceras municipales.

Se muestra en la Fig. 7, la variación del IRCA con respecto a la temporalidad, presentando las gráficas y la distribución mensual del IRCA para aquellos municipios con mayor porcentaje de muestreo ( $\geq 6$ meses), encontrando que para algunos momentos o para algunas temporadas en el año el comportamiento de índice varió drásticamente; además, en la mayoría de los casos el índice no presentó un comportamiento constante $y$, por lo tanto, se asume que puede existir influencia de variables externas. 


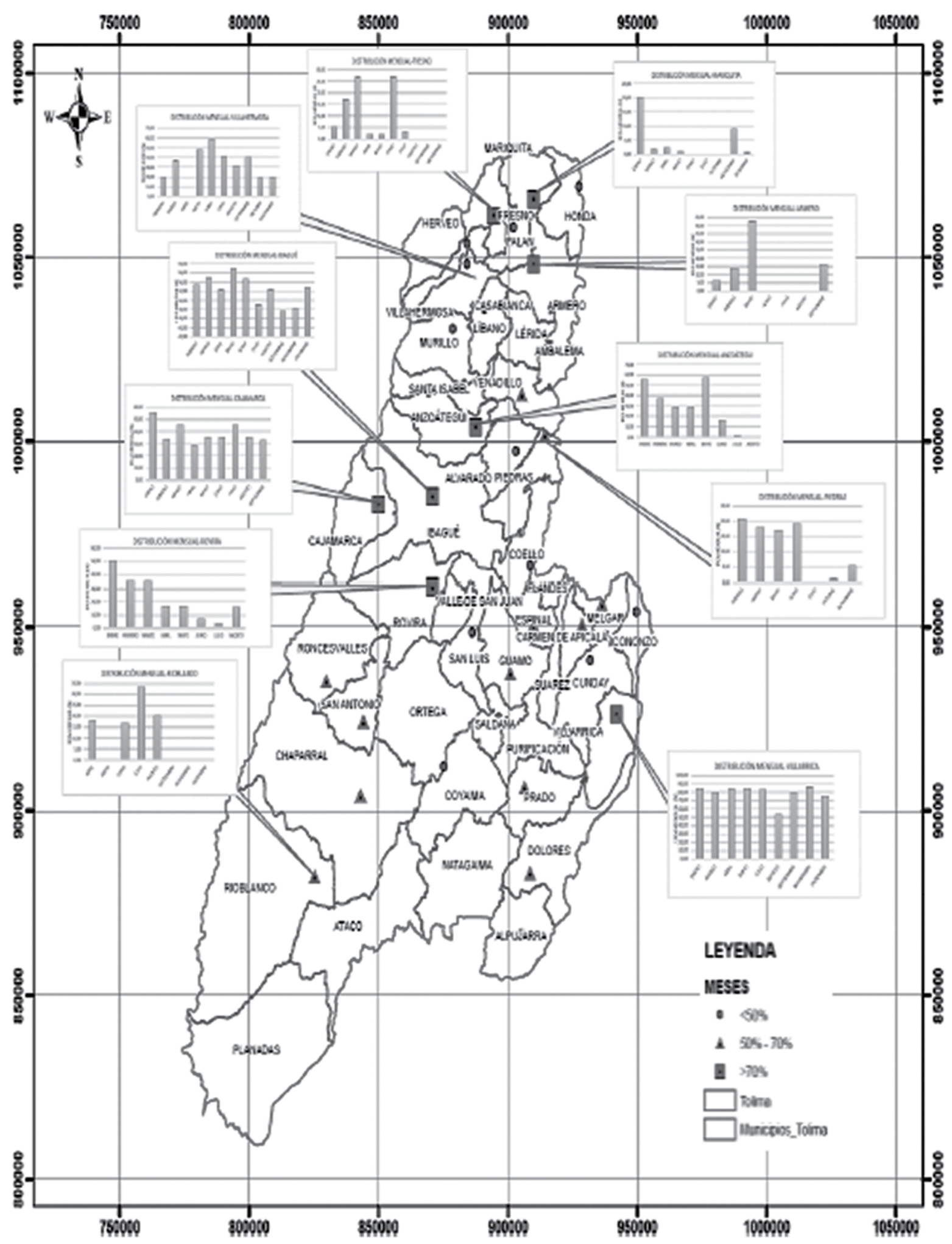

Fig. 7 Mapa de distribución mensual del IRCA en el año 2013. 


\section{Conclusiones y Recomendaciones}

Es claro que aun con la expedición de la Resolución 2115 de 2007 se siguen presentando inconsistencias en la información de la calidad del agua potable reportada en la base de datos del Sivicap, ya que se evidencia la baja calidad de datos respecto de las muestras reportadas, pues se incluyen muestras repetidas, muestras sin reporte de datos y muestras que no poseen medición de parámetros suficientes para el óptimo cálculo del índice; además, alteran el resultado del IRCA y, en este sentido, a las correlaciones, debido a la falta de información consistente que permita identificar razones puntuales para explicar el comportamiento de las variables.

La inclusión de factores externos como el régimen de precipitación y temperatura en la determinación del índice de calidad permitirán establecer medidas de prevención que adviertan sobre el riesgo y que permitan definir criterios adicionales con mayor cobertura teniendo en cuenta el estado de las fuentes abastecedoras, principalmente, cuando se presenten fenómenos anormales como inundaciones, deslizamientos, sequías e incendios forestales.

Los mapas de ubicación de puntos de muestreo con su respectivo nivel de riesgo y distribución mensual de los valores del IRCA representan un análisis puntual y con mayor detalle que propone una comparación objetiva de los resultados del mismo. En efecto, permiten evaluar el comportamiento y la evolución del IRCA en un periodo determinado utilizando elementos en común en todos los años, como lo son los lugares y puntos de muestreo, contribuyendo así a la estructuración de planes y acciones departamentales y municipales que garanticen la mejora continua frente a la calidad del agua para consumo humano.

Los informes nacionales de calidad de agua para consumo humano se basan en la información consolidada y reportada por el Instituto Nacional de Salud - INS en la base de datos del Sivicap. De acuerdo con esto y en referencia a la situación evidenciada frente a las inconsistencias en la información reportada, se concluye que los informes nacionales presentan su análisis basados en información sesgada, que no representa la realidad del IRCA en el departamento; de igual forma, exponen de manera general un histórico del consolidado del IRCA en cada año para un periodo determinado, clasificando a todo el departamento en un nivel de riesgo, cuya validez no se evalúa de forma detallada. Como consecuencia de esto, los programas y subprogramas contemplados en los Planes de Desarrollo Departamental con respecto a la calidad del agua, se estructuran y desarrollan con base en información inconsistente, lo que genera que no se proporcionen los recursos económicos adecuados al departamento para realizar una gestión eficaz que optimice la calidad del agua para consumo humano.

Se recomienda la revisión detallada de factores internos que intervienen en el cálculo del IRCA y la buena operación de la red. Por ejemplo: la selección de los puntos de muestreo, la georreferenciación de estos puntos, frecuencia y métodos de muestreo, cadena de custodia, parámetros analizados e inclusive la capacitación de quienes tienen como función la toma y manejo de las muestras. 


\section{REFERENCIAS}

[1] Ministerio de Salud y Protección Social, Informe Nacional Calidad de Agua para Consumo Humano, Julio de 2007 Diciembre de 2011. Bogotá: Publicaciones Instituto Nacional de Salud, 2013.

[2] Ministerio de la Protección Social. Decreto 1575 de 2007. Bogotá, 2007.

[3] Conpes, Política para el suministro de agua potable y saneamiento básico en la zona rural. Bogotá: Publicaciones Conpes, 2014.

[4] Ministerio de la Protección Social, Resolución 2115 de 2007. Bogotá, 2007.

[5] Gobernación del Tolima, Plan de Desarrollo Unidos por la Grandeza del Tolima 2012 - 2015. Tolima, 2013.

[6] Prasad, T.D; Danso-Amoako, E, 2014. Influence of Chemical and Biological Parameters on Iron and Manganese Acumulation in Water Disribution Networks. Procedia Engineering. ISSN 18777058
[7] Cruz Roja Colombiana - Ideam, Análisis del Cambio Climatico en el país y acciones de la Cruz Roja Colombiana para reducir el riesgo. Bogotá: Publicaciones Ideam, 2009.

[8] Ideam, Estudio Nacional del Agua. Bogotá: Publicaciones Ideam, 2008.

[9] IGAC, Estudio General de Suelos y Zonificación de Tierras Departamento de Tolima. Bogotá: Publicaciones IGAC, 2004.

[10] Universidad del Tolima, Diágnostico General de Tolima. Ibagué: Publicaciones Universidad de Tolima, 2005.

[11] Ideam, Atlas Climatológico de Colombia. Bogotá: Publicaciones Ideam, 2005.

[12] FAO. Papel del ganado doméstico en el control de la desertificación. Publicaciones FAO. Santiago de Chile. 1993. 
